\title{
Ursodeoxycholic acid suppresses epithelial-mesenchymal transition and cancer stem cell formation by reducing the levels of peroxiredoxin II and reactive oxygen species in pancreatic cancer cells
}

\author{
YOON JAE KIM ${ }^{1 *}$, SEOK HOO JEONG ${ }^{2 *}$, EUN-KYUNG KIM ${ }^{1,3}$, EUI JOO KIM ${ }^{1}$ and JAE HEE CHO ${ }^{1}$ \\ ${ }^{1}$ Division of Gastroenterology, Department of Internal Medicine, Gachon University Gil Medical Center, \\ Incheon 21565; ${ }^{2}$ Division of Gastroenterology, Department of Internal Medicine, Catholic Kwandong University, \\ International St. Mary's Hospital, Incheon 22711; ${ }^{3}$ Gachon Medical Research Institute, \\ Gachon University, Gil Medical Center, Incheon 21565, Republic of Korea
}

Received February 25, 2017; Accepted September 28, 2017

DOI: $10.3892 /$ or.2017.6045

\begin{abstract}
Reactive oxygen species (ROS) play a key role in cancer development and progression. Ursodeoxycholic acid (UDCA) may possess antioxidant, anti-inflammatory and chemoprophylatic effects. Therefore, we aimed to investigate the effects and mechanisms of UDCA treatment on pancreatic cancer cells. The pancreatic cancer cell lines HPAC and Capan-1 were treated with 0.2 mM UDCA. To examine alterations in the levels of intracellular ROS, the DCF-DA stain was used and both stemness and epithelial-mesenchymal transition (EMT)-related genes were quantified using qRT-PCR and western blot analysis. The pancreatic cancer sphere culture was performed following seven days of treatment with $0.2 \mathrm{mM}$ UDCA, as an indicator of stemness. Following treatment with UDCA, the level of intracellular ROS was decreased in the pancreatic cancer cells. UDCA decreased both the phosphorylation of STAT3 and the expression of peroxiredoxin II (Prx2). Furthermore, the treatment resulted in the upregulation of E-cadherin and in the downregulation of N-cadherin. In addition, UDCA decreased the expression of sex determining region Y-box 2 (Sox2) and it diminished the number of pancreatic cancer spheres formed. In conclusion UDCA suppressed the levels of intracellular ROS and Prx2 and it decreased EMT and stem cell formation in pancreatic cancer cells. Therefore, UDCA may provide favorable therapeutic benefits, through its antioxidant effects, for patients with pancreatic cancer.
\end{abstract}

Correspondence to: Professor Jae Hee Cho, Division of Gastroenterology, Gachon University, Gil Medical Center, 1198 Guwol-dong, Namdong-gu, Incheon 21565, Republic of Korea E-mail: jhcho9328@gmail.com

${ }^{*}$ Contributed equally

Key words: ursodeoxycholic acid, reactive oxygen species, peroxiredoxin II, epithelial-mesenchymal transition, pancreatic cancer cell

\section{Introduction}

Pancreatic cancer has a generally poor prognosis with a 5-year survival rate of approximately $6 \%$ (1). Pancreatic cancer is the 13th most common cancer and the 4th leading cause of cancer-related deaths in the world (2-4). Since we do not have an effective screening method for pancreatic cancer, most patients are diagnosed at an advanced stage that carries a poor prognosis $(2,5)$. The discovery of molecular alterations in pancreatic cancer has led to the development of targeted therapies that have exhibited substantial benefits in clinical studies, however the survival rate remains poor (6).

Reactive oxygen species (ROS) consist of radicals, ions, or other molecules formed by the reduction of oxygen. The ROS-mediated damage of nucleic acids, proteins and lipids may affect the process of carcinogenesis (7). ROS may influence the cell cycle progression, proliferation, cell survival and apoptosis, angiogenesis and the maintenance of tumor stemness (8). In pancreatic cancer, ROS production is known to be increased. Contrary to other cancer cells, cancer stem cells maintain lower intracellular ROS concentrations (9), which may be the result of decreased ROS production or activated scavenging systems that remove ROS in cancer stem cells $(10,11)$. Oncogenes that affect different pathways have been implicated in increases of ROS production. If oncogenes such as Raf, Myc and cyclin E become overexpressed, ROS production is increased (12). Epithelial-mesenchymal transition (EMT) is a crucial process toward resistance of cell death, chemoresitance, evasion of the immune system, tumor invasion and tumor metastasis $(1,2)$. In some authoritative studies, ROS was suggested as mediators or modulators of the EMT process (3-5). However, the role of ROS in promoting EMT and cancer stem cell formation remains unclear.

Ursodeoxycholic acid (UDCA) is a hydrophilic synthetic bile acid which is the 7- $\beta$-epimer of chenodeoxycholic acid. UDCA is the standard treatment for primary sclerosing cholangitis, primary biliary cirrhosis, cystic fibrosis and intrahepatic cholestasis. Although the effect of UDCA as 
a therapeutic agent in cancer is uncertain, some studies revealed such a possibility. $\mathrm{Xu}$ et al reported that UDCA had an anticancer effect in liver cancer cell lines in vitro and in mice in vivo (13). In addition, recent studies in murine and rat models revealed that UDCA had a chemopreventive effect on colorectal cancer (14-17). UDCA prevented the formation of ROS species and inhibited the Bax protein-translocation from the cytosol to mitochondria in rat liver and human hepatocytes. In addition, anti-apoptotic effects were revealed in other cell types (18-21). However, the anti-carcinogenic mechanisms of UDCA have not been elucidated.

UDCA could play important roles via its anti-apoptotic, anti-inflammatory and chemoprophylactic effects and for this reason, we investigated the mechanisms of action of UDCA in pancreatic cancer cells.

\section{Materials and methods}

Reagents and materials. UDCA was obtained from Sigma-Aldrich (St. Louis, MO, USA) and 2',7'-dichlorofluorescein diacetate $\left(\mathrm{H}_{2} \mathrm{DCF}-\mathrm{DA}\right)$ was purchased from Molecular Probes (Eugene, OR, USA). The antibodies to sex-determining region Y-box 2 (Sox2) (\#4900), E-cadherin (\#3195), N-cadherin (\#13116), anti-rabbit IgG (HRP-linked, \#7074) and anti-mouse IgG (HRP-linked, \#7076) were purchased from Cell Signaling Technology (Beverly, MA, USA). Anti- $\beta$-actin (LF-PA0207) and anti-peroxiredoxin II (Prx2) (LF-MA0144) were obtained from AbFrontier (Seoul, Korea).

Cell lines and culture conditions. The pancreatic cancer cell lines, HPAC and Capan-1 were obtained from Professor Si Young Song (Yonsei University College of Medicine, Seoul, Korea), cultured in a mixture of Dulbecco's modified Eagle's medium (DMEM) and F12 containing 10\% fetal bovine serum (FBS) (HPAC), Iscove's modified Dulbecco's medium (IMDM) containing 20\% FBS (Capan-1) with $1 \%$ penicillin-streptomycin, at $37^{\circ} \mathrm{C}$ in an incubator with a $5 \% \mathrm{CO}_{2}$ atmosphere.

Analysis of ROS by FACS. The level of intracellular ROS was assayed using $\mathrm{H}_{2}$ DCF-DA staining (Thermo Fisher Scientific Inc., Waltham, MA, USA). To assay the ROS level, $1 \times 10^{6}$ cells were seeded in 60-mm dishes (Corning Incorporated, Corning, NY,USA). After $12 \mathrm{~h}$, the seeded cells were treated with $0.2 \mathrm{mM}$ UDCA. After $20 \mathrm{~min}$, the cells were harvested by trypsinEDTA and washed with phosphate buffered saline (PBS). The harvested cells were incubated with $25 \mu \mathrm{M} \mathrm{H}_{2} \mathrm{DCF}-\mathrm{DA}$ at $37^{\circ} \mathrm{C}$ for $30 \mathrm{~min}$. The fluorescence intensity was quantified using flow cytometry (BD Biosciences, Seoul, Korea).

Reverse transcription PCR and quantitative reverse transcription PCR analyses. Total RNA was extracted from harvested cells using an RNeasy Plus Mini kit (Qiagen, Hilden, Germany). To remove genomic DNA, the extracted total RNA was digested by DNase I (New England BioLabs, Beverly, MA, USA). Purified total RNA in the amount of $1 \mu \mathrm{g}$ was reverse transcribed and amplified by RT-PCR using a HighCapacity cDNA Reverse Transcription kit (Applied Biosystems, Foster City, CA, USA). The reactions of quantitative RT-PCR were assessed using SYBR Premix Ex Taq II (Takara, Kusatsu,
Japan) on an iCycler (Bio-Rad Laboratories Inc., Hercules, CA, USA). The following primer pairs were used: human Prx2 (gene ID: 7001), 5'-TGTGATCGTCCGTGCGTCTA-3' and 5'-CCGATGCGCGCGTTAC-3'; human Sox2 (gene ID: 6657), 5'-TGCGAGCGCTGCACAT-3' and 5'-GCAGCGTGTACTT ATCCTTCTTCA-3'; human E-cadherin (gene ID: 999), 5'-CTGAGAACGAGGCTAACG-3' and 5'-GTCCACCATCA TCATTCAATA-3'; human N-cadherin (gene ID: 1000), 5'-TGGATGGACCTTATGTTGCT-3' and 5'-AACACCTGT CTTGGGATCAA-3'; GAPDH (gene ID: 2597), 5'-AGGGCT GCTTTTAACTCTGGT-3' and 5'-CCCCACTTGATTTTGG AGGGA-3'. The thermal conditions for quantitative reverse transcription PCR assay were as follows: cycle $1,95^{\circ} \mathrm{C}$ for $3 \mathrm{~min}$; cycle $2(\mathrm{x} 40)$, at $95^{\circ} \mathrm{C}$ for $10 \mathrm{sec}$ and at $55^{\circ} \mathrm{C}$ for $30 \mathrm{sec}$. Target genes were normalized to GAPDH. The fold change from the untreated control was set at 1-fold, and the normalized fold change ratio was calculated using the $\Delta \Delta \mathrm{Ct}$ method.

Immunoblotting. The collected cells were lysed in cold RIPA lysis buffer (0.5 M Tris-HCl, $\mathrm{pH} 7.4,1.5 \mathrm{M} \mathrm{NaCl}, 2.5 \%$ deoxycholic acid, 10\% NP-40, 10 mM EDTA) with protease and phosphatase inhibitors (GenDepot, Barker, CA, USA). The cell lysates were subjected to SDS-PAGE and transferred onto nitrocellulose membranes (Pall Gelman Laboratory, Ann Arbor, MI, USA). After being blocked with 8\% skim milk or $5 \%$ bovine serum albumin (BSA), the membranes were incubated with primary antibodies overnight at $4^{\circ} \mathrm{C}$. The dilutions used for each antibody were according to the manufacturer's instructions. After being washed in PBS with $0.1 \%$ Tween, the membranes were incubated with a secondary antibody conjugated to horseradish peroxidase. The detection step was performed using WesternBright ECL HRP substrate (Advansta, Menlo Park, CA, USA).

Floating-sphere formation assay. To assay the formation of HPAC and Capan-1 pancreatic cancer spheres, 4,000 cells/well were seeded in 6-well ultralow attachment plates (Corning Incorporated) in serum-free DMEM/F12 (HPAC) or IMDM (Capan-1) with 1X B-27 supplement (50X; Gibco, Invitrogen, Carlsbad, CA, USA), $10 \mathrm{ng} / \mathrm{ml}$ hFGF (\#4114TC-01M; R\&D Systems, Minneapolis, MN, USA), $10 \mathrm{ng} / \mathrm{ml}$ hEGF (\#236-EG-01M; R\&D Systems) and Heparin (Sigma-Aldrich). The plated cells were incubated at $37^{\circ} \mathrm{C}$ in a $5 \% \mathrm{CO}_{2}$ incubator. Seven days after seeding, the pancreatic cancer spheres were counted.

\section{Results}

UDCA suppresses the level of ROS in pancreatic cancer cells through the inhibition of the expression of Prx2 and a reduction of the phosphorylation of STAT3. To detect the antioxidant effect of UDCA in pancreatic cancer cells, we assessed the intracellular ROS levels using DCF-DA staining, as detected by FACS. We found that UDCA decreased the ROS levels in the HPAC and the Capan-1 cells (Fig. 1).

The change of ROS homeostasis by UDCA affected the expression of antioxidant proteins. Prx 2 is an abundant antioxidant protein in cells. To determine whether UDCA affected the expression of Prx2, we assessed the level of Prx 2 using 

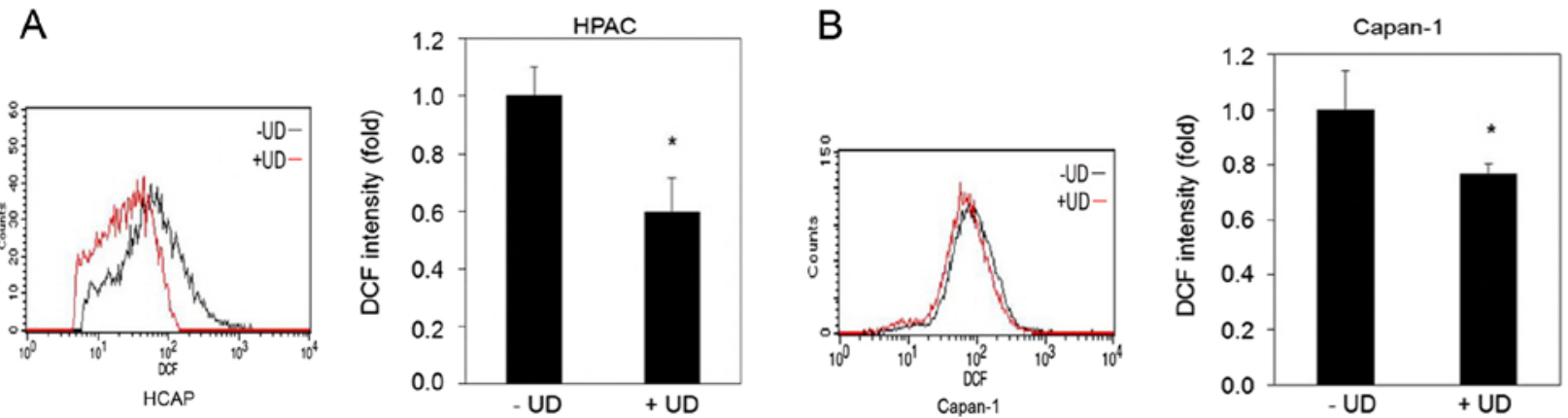

Figure 1. UDCA reduces the level of intracellular ROS in pancreatic cancer cells. (A) HPAC and (B) Capan-1 cells were treated with 0.2 mM UDCA for $20 \mathrm{~min}$ and stained with $25 \mu \mathrm{M}$ DCF-DA for $30 \mathrm{~min}$. The total level of intracellular ROS was detected by FACS analysis. Asterisks indicate statistical significance ( $(\mathrm{p}<0.03)$. UDCA, ursodeoxycholic acid; +UD, cells treated with ursodeoxycholic acid; -UD, cells not treated with ursodeoxycholic acid; ROS, reactive oxygen species.
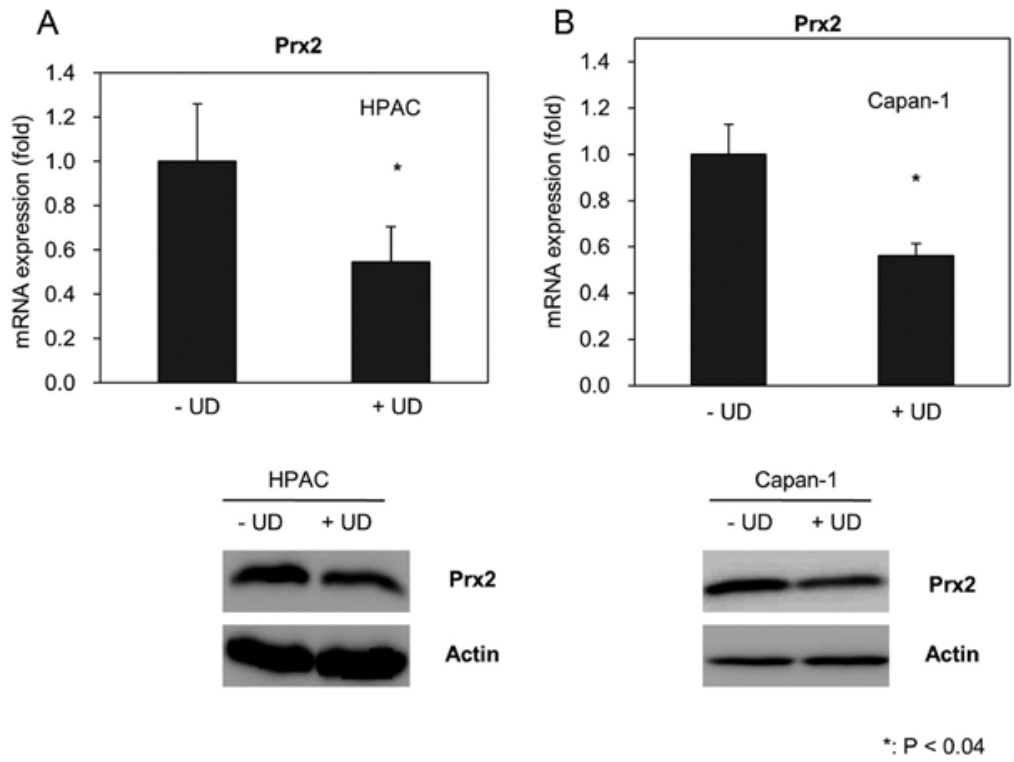

Figure 2. Prx 2 expression is decreased by UDCA. (A) The HPAC cells and (B) the Capan-1 cells were treated with $0.2 \mathrm{mM}$ UDCA for $24 \mathrm{~h}$. The quantification of Prx 2 mRNA was performed by qRT-PCR. The protein expression of Prx 2 was determined by western blotting ("p<0.04). UDCA, ursodeoxycholic acid; $+\mathrm{UD}$, cells treated with ursodeoxycholic acid; -UD, cells not treated with ursodeoxycholic acid; Prx2, peroxiredoxin II.

qRT-PCR and western blotting. UDCA suppressed the production of Prx 2 mRNA in the HPAC and the Capan-1 cells (Fig. 2).

Correspondingly, the protein level of Prx 2 was significantly reduced by UDCA. We observed that treatment of UDCA inhibited the phosphorylation of STAT3 in the HPAC and the Capan-1 cells (Fig. 3).

UDCA decreases EMT in pancreatic cancer cells. UDCA affected the expression of EMT-related proteins. The expression of E-cadherin mRNA was induced by UDCA in HPAC cells (Fig. 4A). Furthermore, the protein level of E-cadherin was increased by UDCA in the HPAC cells. N-cadherin is a representative marker of mesenchymal cells. The expression levels of N-cadherin mRNA and protein were suppressed by UDCA in HPAC cells. The effect of UDCA was replicated in the Capan-1 cells (Fig. 4B). UDCA induced the expression of epithelial marker (ZO-1) in the HPAC and Capan-1 cells. In addition, UDCA suppressed the expression of Slug and ZEB1 in the HPAC cells. The expression of Snail was suppressed by UDCA in Capan-1 cells (Fig. 4C).

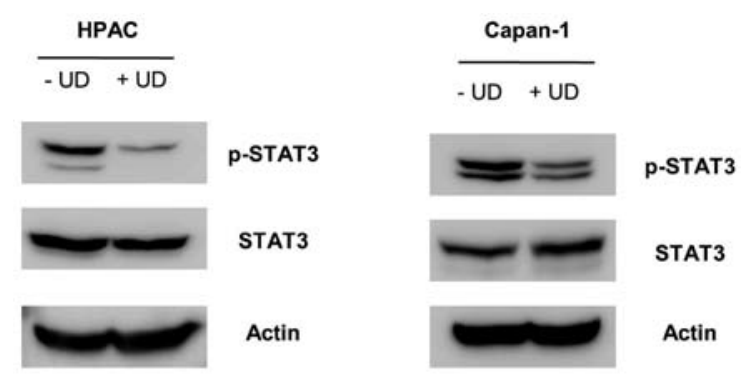

Figure 3. UDCA supresses the phosphorylation of STAT3. The HPAC and the Capan-1 cells were treated with $0.2 \mathrm{mM}$ UDCA for $24 \mathrm{~h}$. The levels of STAT3, phosphorylated STAT3 and $\beta$-actin were detected by western blotting. UDCA, ursodeoxycholic acid; +UD, cells treated with ursodeoxycholic acid; -UD, cells not treated with ursodeoxycholic acid.

UDCA reduces stemness in pancreatic cancer cells. The regulation of Sox 2 is synchronized with Prx2. The expression of Sox 2 mRNA was suppressed by UDCA in the HPAC and Capan-1 cells (Fig. 5A). In addition UDCA reduced the 
A
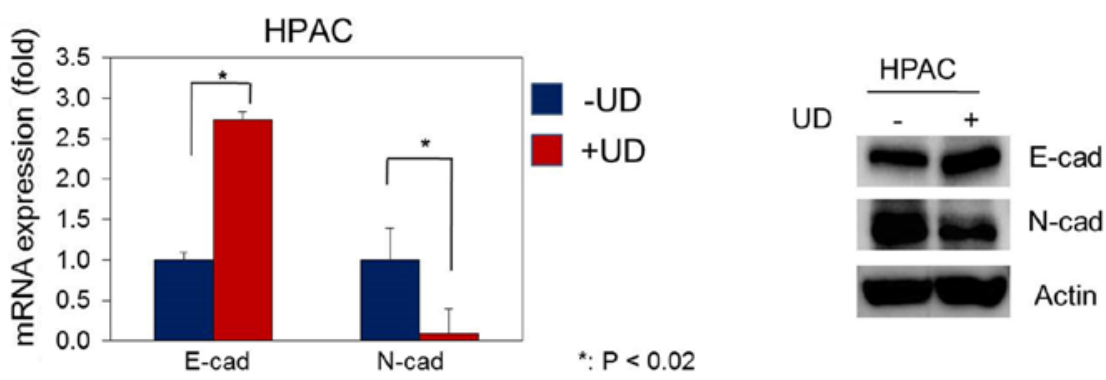

B
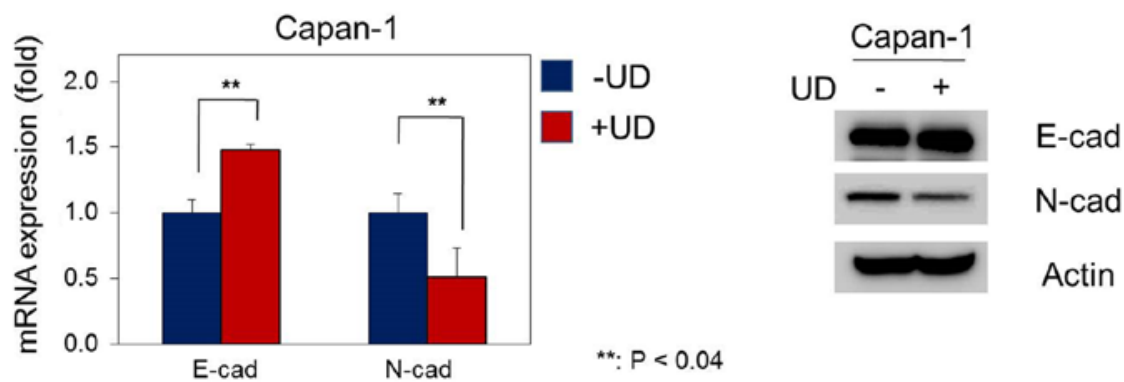

C HPAC

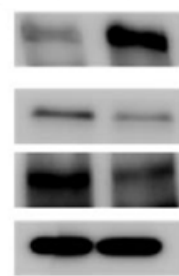

ZO-1

Epithelial marker

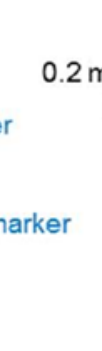

Capan-1

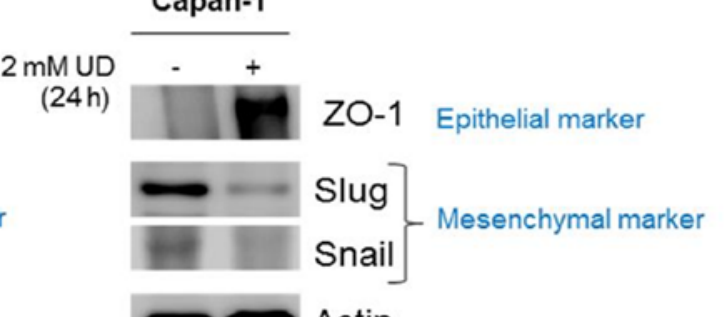

Figure 4. UDCA affects the expression of EMT markers. (A) The HPAC cells and (B) the Capan-1 cells were treated with $0.2 \mathrm{mM}$ UDCA for $24 \mathrm{~h}$. The quantification of E-cadherin and N-cadherin mRNA was accomplished using qRT-PCR. The protein expression of E-cadherin or N-cadherin was determined by western blotting ( $\left.\mathrm{p}<0.02,{ }^{* *} \mathrm{p}<0.04\right)$. (C) The protein expression of the other EMT markers was detected by western blotting after the HPAC and Capan-1 cells had been treated with $0.2 \mathrm{mM}$ UDCA for $24 \mathrm{~h}$. UDCA, ursodeoxycholic acid; +UD, cells treated with ursodeoxycholic acid; -UD, cells not treated with ursodeoxycholic acid; EMT, epithelial mesenchymal transition. E-cad, E-cadherin; N-cad, N-cadherin.

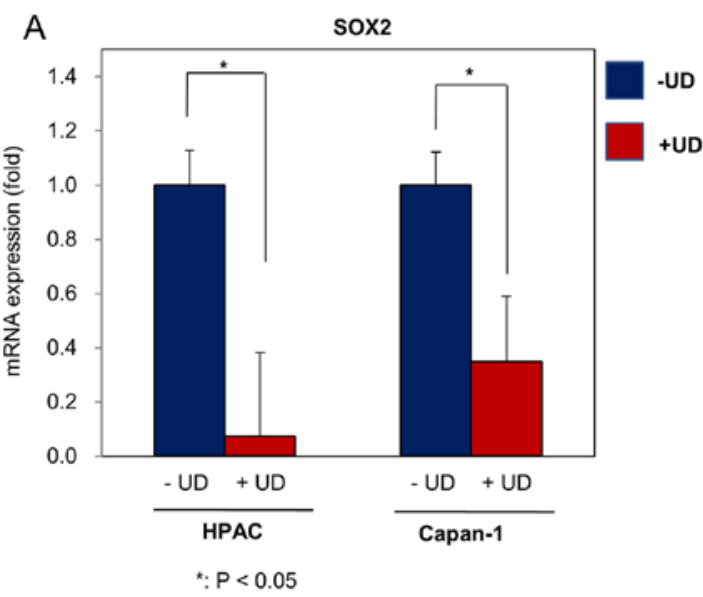

B

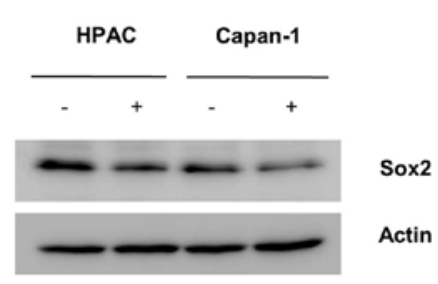

Figure 5. UDCA suppresses the expression of Sox 2 in pancreatic cancer cells. (A) Sox 2 mRNA was quantified using qRT-PCR. The HPAC and Capan-1 cells were treated with $0.2 \mathrm{mM}$ UDCA for $24 \mathrm{~h}(\mathrm{p}<0.05)$. The data are representative of at least 3 replicates. (B) The protein level of Sox 2 was detected by western blotting after the HPAC and Capan-1 cells had been treated with $0.2 \mathrm{mM}$ UDCA for $24 \mathrm{~h}$. UDCA, ursodeoxycholic acid; +UD, cells treated with ursodeoxycholic acid; -UD, cells not treated with ursodeoxycholic acid; Sox2, sex determining region Y-box 2.

protein level of Sox 2 in the HPAC and Capan-1 cells (Fig. 5B). Reduced levels of Sox 2 may affect the formation and growth of cancer stem cells. To investigate whether UDCA reduced the growth of cancer stem cells, we examined the formation 

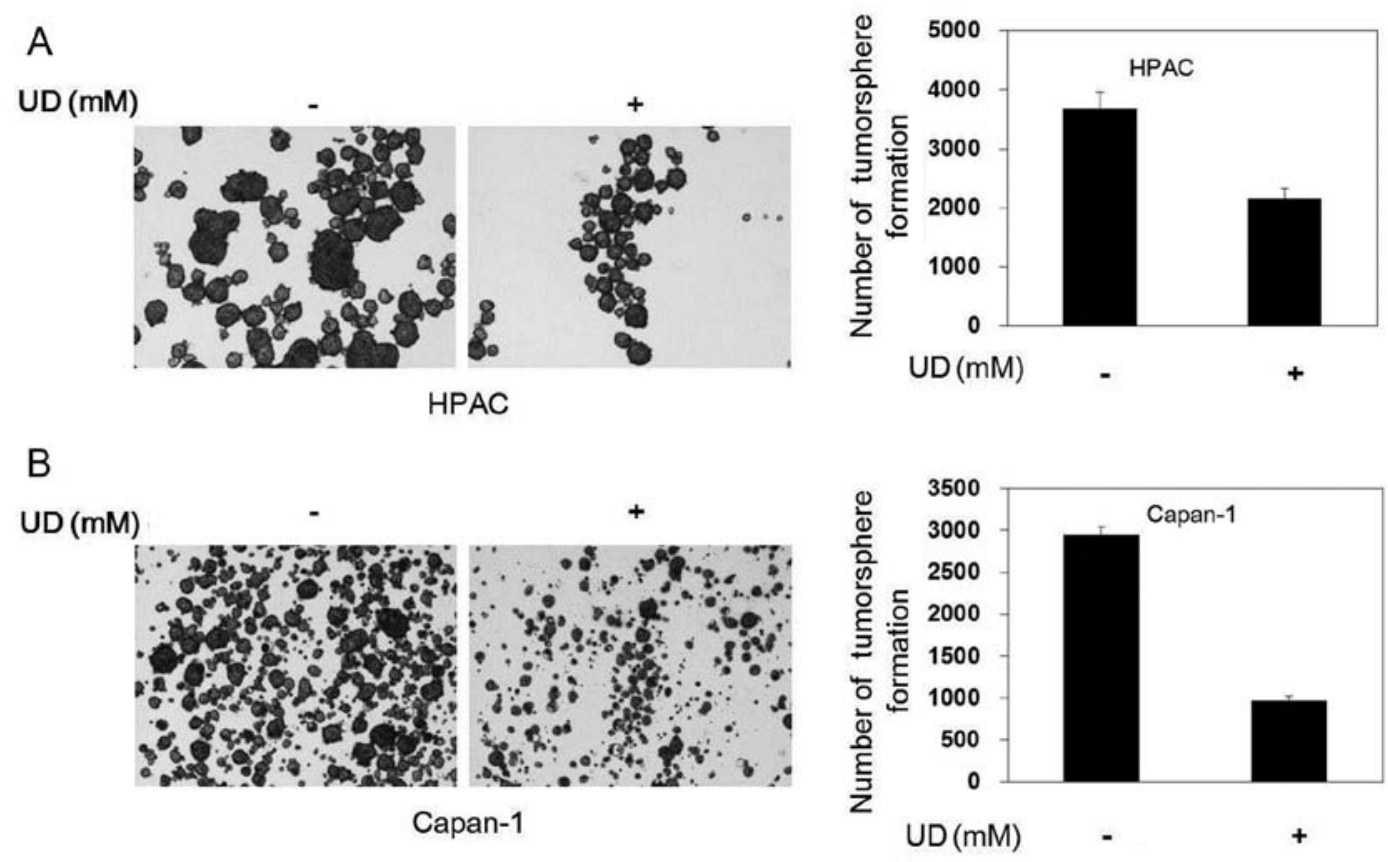

Figure 6. UDCA diminishes the formation of pancreatic tumorspheres. Representative images of tumorspheres in (A) HPAC or (B) Capan-1 cells with or without $0.2 \mathrm{mM}$ UDCA treatment for seven days. All the images were captured with a $4 \mathrm{X}$ objective. Quantification of tumorsphere-forming potential was based upon counting tumorspheres. Error bars denote the standard error $(n=3, p<0.001)$. UD and UDCA, ursodeoxycholic acid.

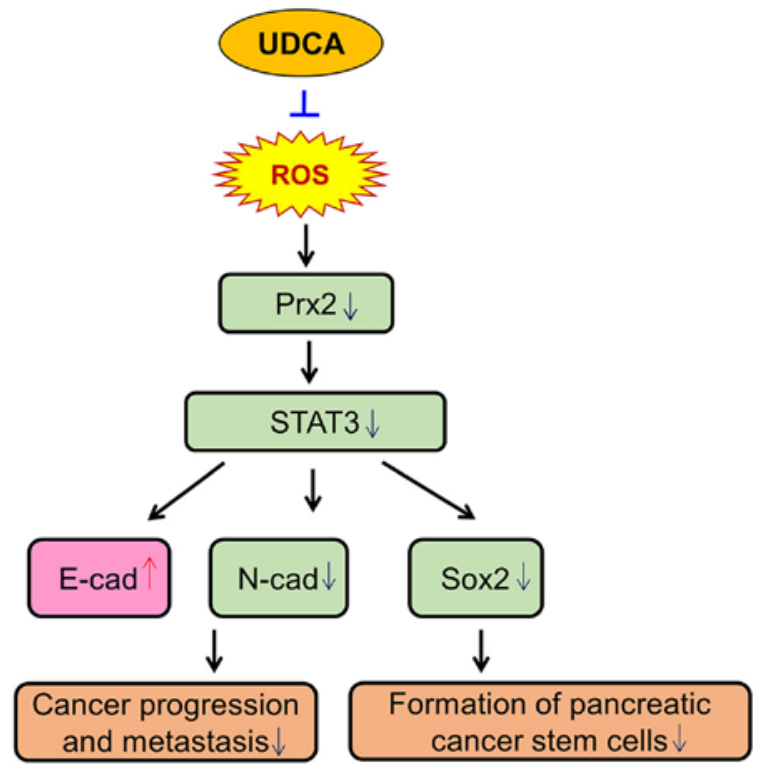

Figure 7. UDCA influences cellular signaling pathways. UDCA reduces the levels of intracellular ROS in pancreatic cancer cells. The reduced level of ROS caused by UDCA leads to a decrease in the interaction between Prx 2 and STAT3 and suppresses phosphorylation of STAT3. The reduction of STAT3 activation caused by UDCA affects the expression of E-cadherin and $\mathrm{N}$-cadherin. The increased expression of E-cadherin and decreased expression of N-cadherin by UDCA affects the formation of cancer stem cells. Attenuation of STAT3 activation influences the expression of Sox 2. The reduction of Sox 2 by UDCA affects the formation of cancer stem cells. UDCA, ursodeoxycholic acid; ROS, reactive oxygen species; Prx2, peroxiredoxin II.

of tumorspheres using culture in ultralow attachment plates. UDCA reduced the size of tumorspheres in the HPAC and Capan-1. In addition the total number of tumorspheres was reduced by UDCA (Fig. 6).

\section{Discussion}

UDCA has multifactorial mechanisms of action. Previous studies (21-24) have revealed that UDCA acts as an inhibitor of Bax protein translocation from the cytosol to mitochondria, exhibits a chemopreventive effect on colorectal cancer, demonstrates anti-apoptotic effects and inhibits the formation of ROS. UDCA is known as an antioxidant and is a well-tolerated drug (18-20). In the present study, UDCA influenced the cellular-signaling pathways (Fig. 7). Both in the HPAC and the Capan-1 cells, UDCA did not affect the same signaling pathway. In the HPAC cells, UDCA did not affect the phosphrylation of Akt, mTOR, ERK and p38. In the Capan-1 cells, UDCA reduced the phosphorylation of Akt and mTOR. However, UDCA did not affect the phosphorylation of ERK and p38 in the Capan-1 cells. Furthermore the present sudy revealed that treatment with UDCA reduced the levels of intracellular ROS in the HPAC and Capan-1 cells (Fig. 1).

The lowered level of ROS influenced the cellular signaling pathways $(25,26)$. The changes in ROS levels can be related to the concentrations of the antioxidant proteins (25,27-29). Prx2 is one of the more abundant proteins able to remove ROS in the cells $(27,30)$. In addition, a recent study revealed that Prx 2 may be associated with drug resistance of cancer stem cells (31). The present study revealed that UDCA reduced the mRNA expression and protein level of Prx 2 in pancreatic cancer cells (Fig. 2). Furthemore, UDCA inhibited the expression of Prx1 (data not shown). The reduced ROS level caused by UDCA did not appear to rely on the induction of antioxidant proteins.

Prx2 interacts with several molecules in a stressed environment $(27,32)$. The altered redox state affects the association between $\operatorname{Prx} 2$ and its interacting proteins $(33,34)$. 
Prx2 interacts with STAT3 following exposure to $\mathrm{H}_{2} \mathrm{O}_{2}$ (35-37). This association generates STAT3 oligomerization and increased phosphorylation of STAT3, which activates the transcriptional function of STAT3. The present study revealed that UDCA reduced the amount of phosphorylated STAT3 (Fig. 3). The reduced level of ROS caused by UDCA led to a decrease in the interaction between Prx 2 and STAT3 and suppressed the phosphorylation of STAT3.

The reduction of STAT3 activation caused by UDCA affected EMT (38-40). The expression of E-cadherin was induced by UDCA treatment in the HPAC and Capan-1 cells (Fig. 4). The expression of N-cadherin was supressed by UDCA in the HPAC and Capan-1 cells (Fig. 4). Thus, the present study revealed that UDCA suppressed EMT in pancreatic cancer cells. However, the HPAC and Capan-1 cell lines may not be sufficient to study the EMT mechanism. Therefore, future experiments using more appropriate cell lines such as circulating tumor cells or more pancreatic cell lines as well as cancer patient samples are needed to improve the study of the EMT mechanism.

Attenuation of the STAT3 activation influenced the expression of Sox 2 (41-43). Recently, a research group revealed that Prx 2 regulated the level of Sox2 (37). As displayed in Fig. 5, the expression of Sox 2 was supressed by UDCA. Sox 2 is one of the essential factors in cancer stem cell pathways. Therefore, the reduction of Sox 2 by UDCA affects the formation of cancer stem cells. In the sphere forming assay, we found that UDCA reduced the formation of pancreatic cancer stem cells in the HPAC and Capan-1 cells (Fig. 6). UDCA also suppressed the maintenance of cancer stem cells in the HPAC and Capan-1 cells (data not shown).

Pancreatic cancer is a health issue worldwide and is one of the most aggressive cancers. Due to this fact novel therapeutic methods are needed for pancreatic cancer, as well as a more thorough understanding of the genetic and molecular pathways involved. The present study supports the potential usefulness of UDCA in pancreatic cancer, due to its antioxidant properties. However, the anticancer mechanism of UDCA has not been fully elucidated. Therefore, future studies are warranted to follow up our results with animal experiments and clinical trials.

In conclusion, UDCA suppressed the level of intracellular ROS and decreased EMT and stem cell formation in a pancreatic cancer cell line. Therefore, the antioxidant effects of UDCA may provide a positive therapeutic benefit for pancreatic cancer patients.

\section{Acknowledgments}

The present study was supported by the Basic Science Research Program through the National Research Foundation of Korea (NRF), funded by the Ministry of Education (nos. NRF-2017R1 D1A1B04034547 and NRF-2017R1D1A1B03034546) and Gachon University Gil Medical Center (2016-15).

\section{References}

1. Knudsen ES, Balaji U, Mannakee B, Vail P, Eslinger C, Moxom C, Mansour J and Witkiewicz AK: Pancreatic cancer cell lines as patient-derived avatars: Genetic characterisation and functional utility. Gut: Jan 10, 2017 (Epub ahead of print). doi: 10.1136/gutjnl-2016-313133.
2. Stathis A and Moore MJ: Advanced pancreatic carcinoma: Current treatment and future challenges. Nat Rev Clin Oncol 7: $163-172,2010$.

3. Siegel R, Naishadham D and Jemal A: Cancer statistics, 2013. CA Cancer J Clin 63: 11-30, 2013.

4. Kleger A, Perkhofer L and Seufferlein T: Smarter drugs emerging in pancreatic cancer therapy. Ann Oncol 25: 1260-1270, 2014.

5. Feng W, Zhang B, Cai D and Zou X: Therapeutic potential of histone deacetylase inhibitors in pancreatic cancer. Cancer Lett 347: 183-190, 2014

6. Wong HH and Lemoine NR: Pancreatic cancer: Molecular pathogenesis and new therapeutic targets. Nat Rev Gastroenterol Hepatol 6: 412-422, 2009.

7. Trachootham D, Alexandre J and Huang P: Targeting cancer cells by ROS-mediated mechanisms: A radical therapeutic approach? Nat Rev Drug Discov 8: 579-591, 2009.

8. Gupta A, Rosenberger SF and Bowden GT: Increased ROS levels contribute to elevated transcription factor and MAP kinase activities in malignantly progressed mouse keratinocyte cell lines. Carcinogenesis 20: 2063-2073, 1999.

9. Shi X, Zhang Y, Zheng J and Pan J: Reactive oxygen species in cancer stem cells. Antioxid Redox Signal 16: 1215-1228, 2012.

10. Diehn M, Cho RW, Lobo NA, Kalisky T, Dorie MJ, Kulp AN, Qian D, Lam JS, Ailles LE, Wong M, et al: Association of reactive oxygen species levels and radioresistance in cancer stem cells. Nature 458: 780-783, 2009.

11. Ishimoto $T$, Nagano $O$, Yae $T$, Tamada M, Motohara $T$, Oshima H, Oshima M, Ikeda T, Asaba R, Yagi H, et al: CD44 variant regulates redox status in cancer cells by stabilizing the $\mathrm{xCT}$ subunit of system $\mathrm{xc}^{-}$and thereby promotes tumor growth. Cancer Cell 19: 387-400, 2011.

12. Sabharwal SS and Schumacker PT: Mitochondrial ROS in cancer: Initiators, amplifiers or an Achilles' heel? Nat Rev Cancer 14: 709-721, 2014.

13. Xu Y, Luo Q, Lin T, Zeng Z, Wang G, Zeng D, Ding R, Sun C, Zhang XK and Chen H: U12, a UDCA derivative, acts as an anti-hepatoma drug lead and inhibits the mTOR/S6K1 and cyclin/CDK complex pathways. PLoS One 9: e113479, 2014.

14. Wali RK, Stoiber D, Nguyen L, Hart J, Sitrin MD, Brasitus T and Bissonnette M: Ursodeoxycholic acid inhibits the initiation and postinitiation phases of azoxymethane-induced colonic tumor development. Cancer Epidemiol Biomarkers Prev 11: 1316-1321, 2002.

15. Loddenkemper C, Keller S, Hanski ML, Cao M, Jahreis G, Stein H, Zeitz M and Hanski C: Prevention of colitis-associated carcinogenesis in a mouse model by diet supplementation with ursodeoxycholic acid. Int J Cancer 118: 2750-2757, 2006.

16. Serfaty L: Chemoprevention of colorectal cancer with ursodeoxycholic acid: Pro. Clin Res Hepatol Gastroenterol 36 (Suppl 1): S53-S60, 2012.

17. van Heumen BW, Roelofs HM, Te Morsche RH, Marian B, Nagengast FM and Peters WH: Celecoxib and tauro-ursodeoxycholic acid co-treatment inhibits cell growth in familial adenomatous polyposis derived LT97 colon adenoma cells. Exp Cell Res 318: 819-827, 2012.

18. Benz C, Angermüller S, Töx U, Klöters-Plachky P, Riedel HD, Sauer P, Stremmel W and Stiehl A: Effect of tauroursodeoxycholic acid on bile-acid-induced apoptosis and cytolysis in rat hepatocytes. J Hepatol 28: 99-106, 1998.

19. Rodrigues CM, Fan G, Ma X, Kren BT and Steer CJ: A novel role for ursodeoxycholic acid in inhibiting apoptosis by modulating mitochondrial membrane perturbation. J Clin Invest 101: 2790-2799, 1998.

20. Benz C, Angermüller S, Otto G, Sauer P, Stremmel W and Stiehl A: Effect of tauroursodeoxycholic acid on bile acidinduced apoptosis in primary human hepatocytes. Eur J Clin Invest 30: 203-209, 2000.

21. Lindor K: Ursodeoxycholic acid for the treatment of primary biliary cirrhosis. N Engl J Med 357: 1524-1529, 2007.

22. Paumgartner G and Beuers U: Ursodeoxycholic acid in cholestatic liver disease: Mechanisms of action and therapeutic use revisited. Hepatology 36: 525-531, 2002.

23. Siegel JL, Jorgensen R, Angulo P and Lindor KD: Treatment with ursodeoxycholic acid is associated with weight gain in patients with primary biliary cirrhosis. J Clin Gastroenterol 37: 183-185, 2003.

24. Perez MJ and Briz O: Bile-acid-induced cell injury and protection. World J Gastroenterol 15: 1677-1689, 2009. 
25. Ray PD, Huang BW and Tsuji Y: Reactive oxygen species (ROS) homeostasis and redox regulation in cellular signaling. Cell Signal 24: 981-990, 2012.

26. Schieber M and Chandel NS: ROS function in redox signaling and oxidative stress. Curr Biol 24: R453-R462, 2014.

27. Rhee SG, Woo HA, Kil IS and Bae SH: Peroxiredoxin functions as a peroxidase and a regulator and sensor of local peroxides. $\mathrm{J}$ Biol Chem 287: 4403-4410, 2012.

28. Poljsak B, Šuput D and Milisav I: Achieving the balance between ROS and antioxidants: When to use the synthetic antioxidants. Oxid Med Cell Longev 2013: 956792, 2013.

29. Espinosa-Diez C, Miguel V, Mennerich D, Kietzmann T, Sánchez-Pérez P, Cadenas S and Lamas S: Antioxidant responses and cellular adjustments to oxidative stress. Redox Biol 6: 183-197, 2015.

30. Wood ZA, Schröder E, Robin Harris J and Poole LB: Structure, mechanism and regulation of peroxiredoxins. Trends Biochem Sci 28: 32-40, 2003

31. Cerda MB, Lloyd R, Batalla M, Giannoni F, Casal M and Policastro L: Silencing peroxiredoxin-2 sensitizes human colorectal cancer cells to ionizing radiation and oxaliplatin. Cancer Lett 388: 312-319, 2017.

32. Latimer HR and Veal EA: Peroxiredoxins in regulation of MAPK signalling pathways; sensors and barriers to signal transduction. Mol Cells 39: 40-45, 2016.

33. Moon JC, Hah YS, Kim WY, Jung BG, Jang HH, Lee JR, Kim SY, Lee YM, Jeon MG, Kim CW, et al: Oxidative stressdependent structural and functional switching of a human 2-Cys peroxiredoxin isotype II that enhances HeLa cell resistance to $\mathrm{H}_{2} \mathrm{O}_{2}$-induced cell death. J Biol Chem 280: 28775-28784, 2005.

34. Qu D, Rashidian J, Mount MP, Aleyasin H, Parsanejad M, Lira A, Haque E, Zhang Y, Callaghan S, Daigle M, et al: Role of Cdk5-mediated phosphorylation of Prx2 in MPTP toxicity and Parkinson's disease. Neuron 55: 37-52, 2007.

35. Park H, Noh AL, Kang JH, Sim JS, Lee DS and Yim M: Peroxiredoxin II negatively regulates lipopolysaccharideinduced osteoclast formation and bone loss via JNK and STAT3. Antioxid Redox Signal 22: 63-77, 2015.
36. Sobotta MC, Liou W, Stöcker S, Talwar D, Oehler M, Ruppert T, Scharf AN and Dick TP: Peroxiredoxin-2 and STAT3 form a redox relay for $\mathrm{H}_{2} \mathrm{O}_{2}$ signaling. Nat Chem Biol 11: 64-70, 2015.

37. Kwon T, Bak Y, Park YH, Jang GB, Nam JS, Yoo JE, Park YN, Bak IS, Kim JM, Yoon DY, et al: Peroxiredoxin II is essential for maintaining stemness by redox regulation in liver cancer cells. Stem Cells 34: 1188-1197, 2016.

38. Hawkins K, Mohamet L, Ritson S, Merry CL and Ward CM E-cadherin and, in its absence, $\mathrm{N}$-cadherin promotes Nanog expression in mouse embryonic stem cells via STAT3 phosphorylation. Stem Cells 30: 1842-1851, 2012.

39. Xiong H, Hong J, Du W, Lin YW, Ren LL, Wang YC, Su WY, Wang JL, Cui Y, Wang ZH, et al: Roles of STAT3 and ZEB1 proteins in E-cadherin down-regulation and human colorectal cancer epithelial-mesenchymal transition. J Biol Chem 287: 5819-5832, 2012.

40. Zhang C, Guo F, Xu G, Ma J and Shao F: STAT3 cooperates with Twist to mediate epithelial-mesenchymal transition in human hepatocellular carcinoma cells. Oncol Rep 33: 1872-1882, 2015.

41. Foshay KM and Gallicano GI: Regulation of Sox 2 by STAT3 initiates commitment to the neural precursor cell fate. Stem Cells Dev 17: 269-278, 2008.

42. Gao H, Teng C, Huang W, Peng J and Wang C: SOX2 Promotes the epithelial to mesenchymal transition of esophageal squamous cells by modulating slug expression through the activation of STAT3/HIF- $\alpha$ Signaling. Int J Mol Sci 16: 21643-21657, 2015.

43. Wang H, Cai HB, Chen LL, Zhao WJ, Li P, Wang ZQ and Li Z: STAT3 correlates with stem cell-related transcription factors in cervical cancer. J Huazhong Univ Sci Technolog Med Sci 35: 891-897, 2015. 